\title{
High-frequency oscillations: The state of clinical research
}

\author{
*Birgit Frauscher, $\uparrow$ Fabrice Bartolomei (D), $\ddagger$ Katsuhiro Kobayashi, §Jan Cimbalnik, \\ qMaryse A. van 't Klooster, \#Stefan Rampp, **Hiroshi Otsubo, †† Yvonne Höller,

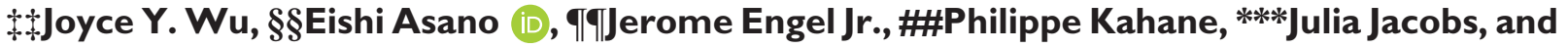 \\ ††'Jean Gotman
}

\author{
Epilepsia, 58(8):1316-1329, 2017 \\ doi: 10.1111/epi.13829
}

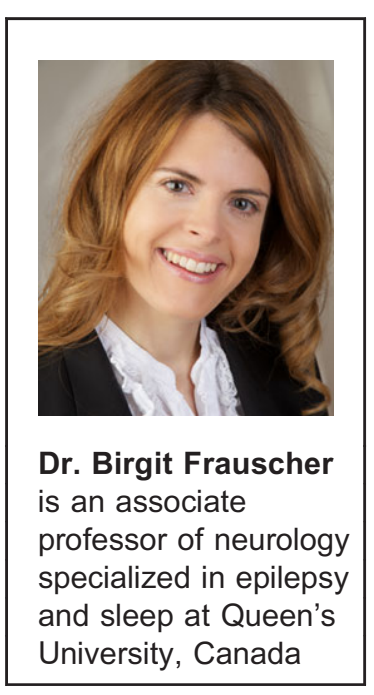

\begin{abstract}
SUMMARY
Modern electroencephalographic (EEG) technology contributed to the appreciation that the EEG signal outside the classical Berger frequency band contains important information. In epilepsy, research of the past decade focused particularly on interictal high-frequency oscillations (HFOs) $>80 \mathrm{~Hz}$. The first large application of HFOs was in the context of epilepsy surgery. This is now followed by other applications such as assessment of epilepsy severity and monitoring of antiepileptic therapy. This article reviews the evidence on the clinical use of HFOs in epilepsy with an emphasis on the latest developments. It highlights the growing literature on the association between HFOs and postsurgical seizure outcome. A recent meta-analysis confirmed a higher resection ratio for HFOs in seizure-free versus non-seizure-free patients. Residual HFOs in the postoperative electrocorticogram were shown to predict epilepsy surgery outcome better than preoperative HFO rates. The review further discusses the different attempts to separate physiological from epileptic HFOs, as this might increase the specificity of HFOs. As an example, analysis of sleep microstructure demonstrated a different coupling between HFOs inside and outside the epileptogenic zone. Moreover, there is increasing evidence that HFOs are useful to measure disease activity and assess treatment response using noninvasive EEG and magnetoencephalography. This approach is particularly promising in children, because they show high scalp HFO rates. HFO rates in West syndrome decrease after adrenocorticotropic hormone treatment. Presence of HFOs at the time of rolandic spikes correlates with seizure frequency. The time-consuming visual assessment of HFOs, which prevented their clinical application in the past, is now overcome by validated computer-assisted algorithms. HFO research has considerably advanced over the past decade, and use of noninvasive methods will make HFOs accessible to large numbers of patients. Prospective multicenter trials are awaited to gather information over long recording periods in large patient samples.

KEY WORDS: Scalp EEG, Biomarker, Surgical outcome, Seizure, Sleep.
\end{abstract}

Accepted May 25, 2017; Early View publication June 30, 2017.

*Department of Medicine and Center for Neuroscience Studies, Queen’s University, Kingston, Ontario, Canada; †National Institute of Health and Medical Research, Institute of Neurosciences of Systems, Aix Marseille University, Marseille, France; \$Department of Child Neurology, Okayama University Graduate School of Medicine, Dentistry and Pharmaceutical Sciences, Okayama University Hospital, Kita-ku, Okayama, Japan; §International Clinical Research Center, St. Anne's University Hospital, Brno, Czech Republic; qDepartment of Neurology and Neurosurgery, Brain Center Rudolf Magnus, University Medical Center Utrecht, Utrecht, The Netherlands; \#Department of Neurosurgery, University Hospital Erlangen, Erlangen, Germany; **Division of Neurology, Department of Pediatrics, Hospital for Sick Children, Toronto, Ontario, Canada; ††Department of Neurology, Christian Doppler Medical Center and Center for Cognitive Neuroscience, Paracelsus Medical University Salzburg, Salzburg, Austria; $\$$ t†Division of Pediatric Neurology, Mattel Children's Hospital at UCLA, Los Angeles, California, U.S.A.; $\S \S$ Departments of Pediatrics and Neurology, Detroit Medical Center, Children's Hospital of Michigan, Wayne State University, Detroit, Michigan, U.S.A.; qףDepartments of Neurology, Neurobiology, and Psychiatry, Brain Research Institute, University of California, Los Angeles, Los Angeles, California, U.S.A.; \#\#Department of Neurology, GrenobleAlpes University Hospital and Grenoble-Alpes University, Grenoble, France; ***Department of Neuropediatrics and Muscular Diseases, University Medical Center Freiburg, Freiburg, Germany; and $\dagger \dagger$ Montreal Neurological Institute and Hospital, McGill University, Montreal, Quebec, Canada

Address correspondence to Birgit Frauscher, MD, Department of Medicine and Centre for Neuroscience Studies, Queen's University, Botterell Hall, 18 Stuart Street, Kingston K7L 3N6, ON, Canada. E-mail: birgit.frauscher@queensu.ca

J.J. and J.G. share senior authorship.

Wiley Periodicals, Inc.

(C) 2017 International League Against Epilepsy 


\section{Key Points}

- This article reviews the evidence on the clinical use of HFOs in epilepsy

- The application of HFOs ranges from epilepsy surgery to the assessment of epilepsy severity and monitoring of antiepileptic therapy

- A recent meta-analysis confirms a higher resection ratio for HFOs in seizure-free versus non-seizure-free patients

- The association with sleep features is used to separate physiological from pathological HFOs to increase the specificity of HFOs

- HFOs seem to be useful to measure disease activity and assess treatment response using noninvasive EEG and MEG

Modern electroencephalographic (EEG) technology has substantially contributed to the appreciation that the EEG signal outside the classical Berger frequency band ranging from 0.3 to $70 \mathrm{~Hz}$ contains important information. In epilepsy, research in recent years has focused particularly on interictal high-frequency oscillations (HFOs) $>80 \mathrm{~Hz}$. HFOs are defined as spontaneous EEG events in the frequency range between 80 and $500 \mathrm{~Hz}$, consisting of at least four oscillations that clearly stand out from the background activity. ${ }^{1}$ HFOs are subdivided into ripples ranging from 80 to $250 \mathrm{~Hz}$ and fast ripples $>250 \mathrm{~Hz} .^{2}$ For more details on the definition of HFOs, see the accompanying article in this issue by Zijlmans et al. $^{3}$ on how to record HFOs. The first large application of HFOs was in the context of epilepsy surgery. This is now followed by other applications such as assessment of epilepsy severity or monitoring of antiepileptic treatment response using noninvasive methodology. This article reviews the latest developments relevant for the clinical use of HFOs and discusses the implications of HFO assessment for clinical epilepsy care.

\section{Milestones in human HFO research}

In 1992, high-frequency (HF) activity in the EEG was first investigated at seizure onset. ${ }^{4,5}$ Independently, in 1999, a different type of brief interictal fast oscillatory event up to $500 \mathrm{~Hz}$ was discovered by the group of Bragin and Engel at the University of California, Los Angeles in animals with experimental epilepsy. At the same time, they also pioneered the recording of HFO events in therapy-refractory patients with mesiotemporal lobe epilepsy. ${ }^{2,6,7}$ In humans, Bragin et al. used microelectrode arrays consisting of 9-18 microwires $($ diameter $=40 \mu \mathrm{m})$ that extended beyond the tip of clinical depth electrodes and recorded from the hippocampus and entorhinal cortex. The authors found ripples $(80-250 \mathrm{~Hz})$ - similar to those described in experimental animals - and fast ripples $(>250 \mathrm{~Hz})$, which are found only in the epileptic condition. ${ }^{2,6}$ This group further characterized microelectrode recordings of human HFOs in the entorhinal cortex ${ }^{8}$ and during different stages of sleep. ${ }^{9}$

The breakthrough in clinical HFO research was made at the Montreal Neurological Institute (MNI) in 2006. Gotman's group showed that HFOs up to $500 \mathrm{~Hz}$ could be recorded using macroelectrodes. In their work, the authors used the MNI electrode with a surface of $1 \mathrm{~mm}^{2} .{ }^{10,11}$ Otsubo's group in Toronto had also demonstrated activity in the $60-150-\mathrm{Hz}$ range in the electrocorticogram of children at the time of spasms. ${ }^{12}$ The detection of HFOs with macroelectrodes resulted in multiple studies by different groups around the world using commercial macroelectrodes as well as subdural electrodes with up to $7 \mathrm{~mm}^{2}$ surface. ${ }^{1}$ Although it was shown that the electrode contact size, within a certain range, does not influence the detectability of HFOs, ${ }^{13}$ it is still unclear whether HFOs assessed with microelectrodes represent the same phenomenon as HFOs detected with macroelectrodes. Interestingly, ripples recorded with macroelectrodes seem to be more localizing than ripples recorded with microelectrodes, and currently there is insufficient explanation for this situation.

The next milestone was the detection of HFOs using noninvasive modalities. The pioneers in this area were Kobayashi's group at Okayama University, which reported ripple activity in the surface EEG of children with electrical status epilepticus during slow wave sleep, ${ }^{14}$ and Gotman's group at the MNI in the surface EEG of adults with focal epilepsy. ${ }^{15}$ Only recently, the first promising pilot studies in magnetoencephalography (MEG) for the detection of HFOs were published. ${ }^{16-18}$ These developments open a new avenue to apply HFOs for the evaluation of different questions, including the assessment of disease activity, to large numbers of patients.

\section{HFOS AS MARKERS OF THE EPILEPTOGENIC ZONE}

\section{Interactions between HFOs and epileptic spikes}

HFOs are often observed at the time of epileptic spikes. Urrestarazu et al. ${ }^{19}$ described three different patterns: (1) $64 \%$ occurred together with spikes and were visible as riding on the spike in the unfiltered signal, (2) 17\% occurred together with spikes but were invisible in the unfiltered spikes, and (3) 19\% occurred completely independently of spikes in timing and localization.

Despite this frequent co-occurrence, there is now sufficient evidence that HFOs and spikes represent different neurophysiological mechanisms. Rodent epilepsy models led to two main findings: (1) HFOs and spikes can occur independently of each other and show separate modulations during the period of epileptogenesis, and (2) epileptic spikes with or without HFOs also seem to have distinct pathophysiological relevance and occur over different brain regions (see the accompanying paper by Jiruska et al. for more 
details $^{20}$ ). Furthermore, HFOs seem to increase before the occurrence of seizures, ${ }^{10,21,22}$ whereas spikes are more prominent postictally. $^{23,24}$

In general, HFOs were shown to be more specific for the seizure-onset zone (SOZ) than spikes. ${ }^{1}$ Applying a threshold with $95 \%$ specificity, the highest sensitivity for identification of the SOZ was found for fast ripples (52\%), followed by ripples (38\%) and spikes alone (33\%). ${ }^{25}$ Interestingly, ripples co-occurring with a spike were shown to be even more closely related to the SOZ than ripples without a spike. $^{26}$

It is probable that some HFOs result from filtering spikes, ${ }^{27}$ but it is clear that a large proportion do not. Van Klink et al. ${ }^{28}$ found that $64 \%$ of ripples started on average $10 \mathrm{~ms}$ before the onset of the spike. They concluded that ripples are therefore unlikely to result from spikes. Jacobs et al. ${ }^{29}$ demonstrated that the HF power increase during spikes is less specific for the SOZ than visually identified individual HFO events. It was further shown that for clinical application, it may not be necessary to separate real HFOs from "false oscillations" produced by the filter effect of sharp spikes. ${ }^{30}$ Burnos et al. classified ripples based on their morphology, with types defined according to regularity in amplitude and frequency: type 1 with regular amplitude and frequency; type 2 with irregular amplitude, which could result from filtering of sharp spikes; type 3 with irregular frequency; and type 4 with irregular amplitude and frequency. The authors found that all types were significantly higher inside the SOZ than outside the SOZ. ${ }^{30}$ Spikes and HFOs are likely to be distinct events; their common cooccurrence might therefore be used in the clinical application to identify the subset of epileptic spikes most closely related to the $\mathrm{SOZ}$.

\section{Interactions between HFOs and seizures}

Tonic HF activity typically in the beta and gamma frequency range at seizure onset has to be differentiated from ictally occurring isolated brief HFO events, which will be discussed in this review. HFOs were shown to increase only immediately prior to or at seizure onset. ${ }^{10,21}$ When examining HFO fluctuations in the 15-, 5-, and 1-min intervals preceding seizure occurrence, no systematic change was found. ${ }^{31}$ Experimental research in the animal model of mesiotemporal lobe epilepsy demonstrated that specific HFO patterns are associated with different seizure-onset patterns; ripples $(>80 \mathrm{~Hz})$ predominate during low-voltage fast activity seizures, whereas fast ripples $(>250 \mathrm{~Hz})$ predominate during periodic spiking seizures (for further information see the paper by Jiruska et al. on experimental insights on HFOs in this issue $\mathrm{e}^{20}$ ).

Sato et al. ${ }^{32}$ showed that there is a relative power reduction of the postspike slow wave relative to the increase of spike-related HFOs in the SOZ immediately preceding ictal onset. Particularly in the SOZ, this correlation is drastically reduced during the 3 -min period preceding seizure onset. ${ }^{33}$
Whereas HFOs remain confined to the same possibly epileptogenic area during interictal and ictal periods, spikes are more widespread during seizures than interictally. ${ }^{34}$ Akiyama et al. investigated the relationship between ictal HFOs and semiologic progression from electrographic ictal onset to clinical manifestation. They found that the ictal HFO propagation corresponded to the ictal semiology in Jacksonian seizures. ${ }^{12,35}$ Usui et al. ${ }^{36}$ investigated ictal very fast HFOs exceeding $1,000 \mathrm{~Hz}$ in 13 patients with intractable epilepsy who underwent epilepsy surgery. Six of the seven patients with ictal very fast HFOs had a favorable outcome, compared to one of six patients with an unfavorable surgical outcome.

The increase in faster frequencies between 60 and $100 \mathrm{~Hz}$ at ictal onset was used to develop a method allowing statistical images of HFOs coregistered with the patient's magnetic resonance imaging (MRI) to further explore the relationships between ictal and interictal HFOs, and to compare these data with other localizing methods. ${ }^{37}$ Currently no final conclusion can be drawn regarding the relative clinical relevance of ictal or interictal HFOs for the identification of the epileptogenic zone.

\section{Resection of areas with high HFO rates is associated with a good postsurgical seizure outcome}

Retrospective studies using depth electrodes, grids, or intraoperative corticography showed that resection of areas with presurgical high HFO rates is associated with a better postsurgical seizure outcome than resection of areas with presurgical low HFO rates. ${ }^{1,38-45}$ Importantly, it was recently shown by Zijlmans' group using intraoperative electrocorticography that the rate of presurgically measured HFOs did not predict seizure outcome, but that the rate measured after resection did. ${ }^{46,47}$ This suggests that it is critical to disconnect networks generating HFOs rather than remove all areas that generate HFOs prior to surgery. ${ }^{47}$ It might also explain the negative association with postoperative seizure outcome in some patients, in whom HFO rates were assessed in presurgical chronic intracranial EEG recordings. An example of a patient who had residual fast ripples in the postsurgical electrocorticogram and who was not seizurefree after surgery is given in Figure 1.

A meta-analysis from 2015 investigated the existing evidence for the relation between resection of HFO-generating regions and outcome after epilepsy surgery. Höller et al. ${ }^{48}$ analyzed the probability that a patient, who is seizure-free after surgical intervention, had a high HFO resection ratio. The authors defined the HFO ratio as the ratio of the number of channels with HFOs that were inside the resected area to the number of channels on which HFOs were detected. Rates of both ripples and fast ripples were shown to have a significant effect: a higher resection ratio for HFOs was found in seizure-free versus non-seizure-free patients. The total effect size, however, was small for both ripples and fast ripples (Fig. 2). The clinically important question of 

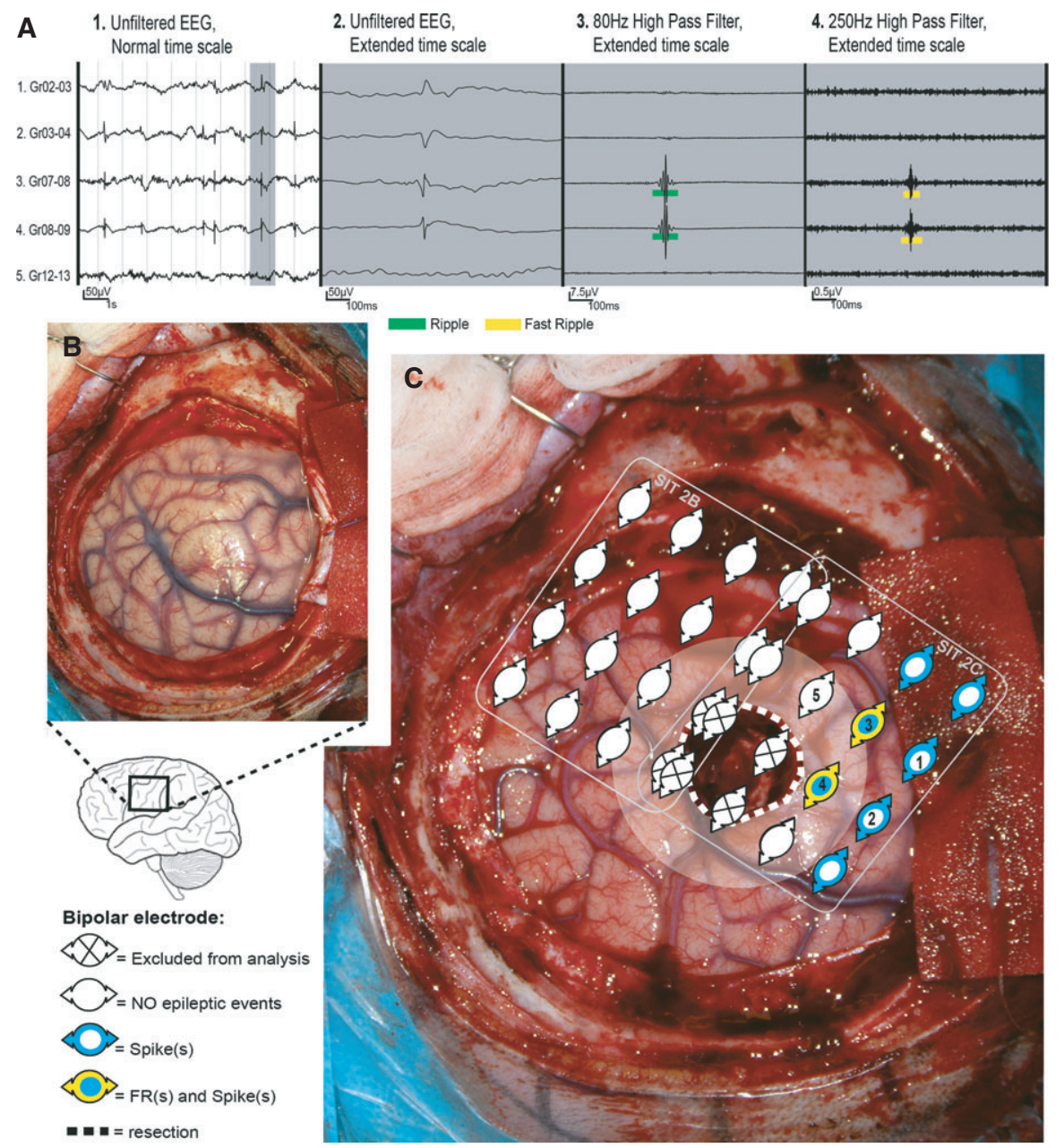

Figure I.

Patient with a left central lesion, with fast ripples (FRs) in post-electrocorticography (ECoG) and recurrent seizures (auras) after surgery. (A) Spike and high-frequency oscillation events in a selection of bipolar channels (indicated as I-5 in C). (B) Preresection photograph. (C) Post-ECoG. The resected area is delineated by the dotted white line. The area near the resection $($ resection margin $=\mathrm{I} \mathrm{cm}$ ) is marked with transparent white. Two ECoG recordings were performed postresection. We represent the location of the bipolar channels analyzed. Note that FRs (rate $=25 / \mathrm{min} /$ electrode; yellow) were present in the margin of the resected lesion (dysembryoplastic neuroepithelial tumor), in a larger region with spikes (range rate $=4-4 \mathrm{l} / \mathrm{min} /$ electrode; blue). Almost all electrodes showed ripples and are therefore not depicted. Based on the FRs present in the resection margin, a different surgical decision could have been made if FRs were not as close to eloquent central cortex. EEG, electroencephalogram. Source: van 't Klooster et al., ${ }^{46}$ with permission from Wolters Kluwer.

Epilepsia C ILAE

whether a patient will become seizure-free if the resection ratio is high cannot be answered by the existing data.

In contrast to this growing evidence from interictal HFOs, there is only sparse evidence on the association of ictal HFOs and postsurgical seizure freedom. A Cochrane review ${ }^{49}$ assessing this question identified two studies with a total of 11 participants who implemented identification of ictal HFOs in their surgical decision making. The authors concluded that no reliable conclusions could be drawn regarding the efficacy of HFO recordings in epilepsy surgery decision making, due to methodological limitations and the small sample size. ${ }^{49}$

One has to differentiate between the SOZ and the epileptogenic zone (the area of brain necessary and sufficient for spontaneous seizures to occur). Interictal markers (such as spikes or HFOs) are only partially effective at localizing the $\mathrm{SOZ}$, but this correspondence is of secondary importance, because we want to identify the epileptogenic zone. The $\mathrm{SOZ}$ is not a perfect marker of the epileptogenic zone; the region where seizures actually start is not necessarily the region where seizures can start. If the $\mathrm{SOZ}$ was a perfect biomarker, people undergoing epilepsy surgery in whom the SOZ was removed should be seizure-free, and clinical practice shows that this is not the case. Therefore, there is a need to develop biomarkers for the epileptogenic zone apart from the SOZ, and HFOs could be one such marker to improve the approximation of the epileptogenic zone with the ultimate aim of improving surgical outcome in people 


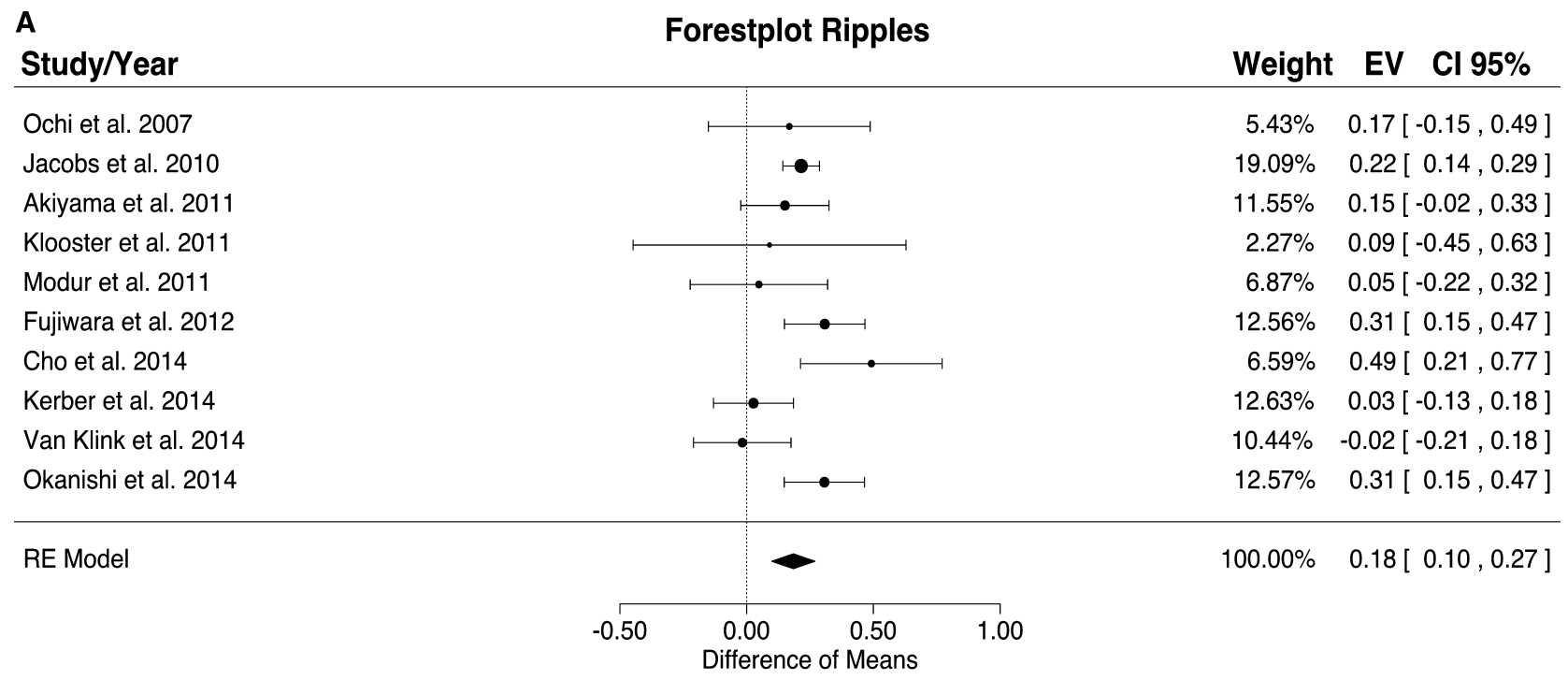

B

Study/Year

Forestplot Fast Ripples

\section{Weight EV Cl 95\%}

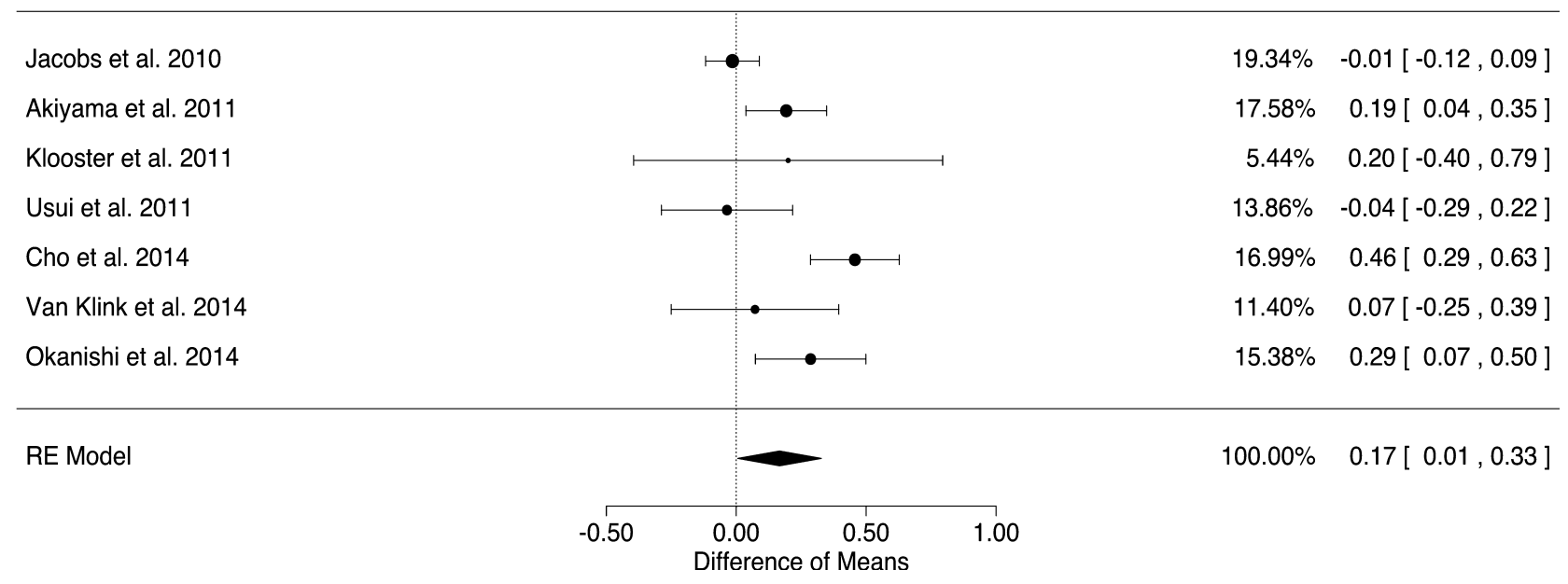

Figure 2.

Meta-analysis results for ripples (A) and fast ripples (B). The resection ratio for both ripples and fast ripples is higher in seizure-free patients compared to non-seizure-free patients. For each study, a graphical representation of the effect (i.e., the difference of the resection ratio between the good- and bad-outcome groups) and of the confidence interval $(\mathrm{Cl})$ is given along with the exact values (EVs) and the weights. RE, rodent epilepsy. Source: Höller et al., ${ }^{48}$ published open access using a Creative Commons Attribution CC-BY licence.

Epilepsia (C) ILAE

with epilepsy. Level I evidence on the clinical use of interictal and ictal HFOs for delineation of the epileptogenic zone and outcome prognosis during epilepsy surgery will require a prospective randomized controlled trial approach (see the section Future Directions). Main challenges for the feasibility of such a prospective trial to prove superiority of HFOs over spikes and the SOZ to improve the surgical success rate are the large sample size needed, requiring international collaboration, standardization of the analysis of HFOs (see the section Visual versus Automatic Detection of HFOs), and differentiation between physiological and pathological
HFOs (see the section Differentiation between Physiological and Pathological HFOs).

\section{HFOs mirror disease activity}

The first evidence that HFOs mirror disease activity comes from experimental work. Bragin et al..$^{50}$ found in the kainic acid model of temporal lobe epilepsy a significant inverse relation between the time of the first HFO detection and the subsequent rate of spontaneous seizures. Later, a study in human intracranial EEG showed that HFOs, in contrast to spikes, do not increase after seizures but increase 
after medication reduction, similarly to seizures. ${ }^{51}$ This implies that spikes and HFOs have different pathophysiologic mechanisms and that HFOs are more tightly linked to seizures than spikes. HFOs therefore can be a useful clinical marker for disease activity. The hypothesis that the more HFOs are generated by the tissue, the higher the seizure frequency was not confirmed, although there might be a correlation for high fast ripple rates. Propofol, known for its antiepileptic effects, was shown to reduce the number of epileptic HFOs, ${ }^{52}$ whereas etomidate, a short-acting anesthetic, activates epileptic HFOs. Importantly, the spatial distribution of these activated HFOs did not extend beyond electrodes showing HFOs without etomidate. ${ }^{53}$ The hypothesis that in epilepsy HFOs mirror disease activity was confirmed in patients with focal cortical dysplasia. HFO rates were higher in patients with focal cortical dysplasia type II lesions compared to type I lesions; usually type II lesions are more epileptogenic with an earlier onset of seizures as well as a higher seizure frequency. ${ }^{54}$

Following this concept, HFOs might also be useful for monitoring antiepileptic drug treatment. ${ }^{55}$ Moreover, studies have shown that HFO rate correlates with disease severity, HFO rate decreases during immunomodulatory therapy, and $\mathrm{HFO}$ rate is able to predict the course of disease (see the section Important Aspects of HFOs in Children). ${ }^{56,57}$

\section{Contribution of noninvasive methods for measuring epileptogenicity}

\section{Scalp EEG}

HF activity was first reported at the onset of epileptic spasms on scalp recordings in children, ${ }^{58,59}$ as well as at the onset of tonic seizures in Lennox-Gastaut syndrome. ${ }^{60}$ More importantly, the first studies on scalp interictal HFO events $(70-200 \mathrm{~Hz})$ were published in children with electrical status epilepticus during slow wave sleep,${ }^{14}$ as well as in idiopathic partial epilepsies of childhood. ${ }^{61}$ The first study on interictal HFOs in adults with focal epilepsy was published in $2011 .{ }^{15}$ An example of scalp HFOs as seen in the unfiltered EEG, the filtered EEG $>80 \mathrm{~Hz}$, and the time-frequency plot is given in Figure 3.

Detection of HFOs in the scalp EEG was at first glance surprising, given the small HFO generator size of 100$200 \mu \mathrm{m}$ and the postulated necessary cortical activation area of $10 \mathrm{~cm}^{2}$ for generating a signal visible on the scalp. A recent simulation study, however, challenged this notion and showed that fast oscillations can be detected within the low noise level of the ripple band $(80-200 \mathrm{~Hz})$ although their median amplitude on scalp EEG recordings is $>10$ times smaller than that of interictal epileptiform discharges and consistent with cortical generators of approximately $1 \mathrm{~cm}^{2}{ }^{62}$ A study using data from simultaneous scalp EEG and intracranial recording confirmed the findings of the latter simulation study and demonstrated that scalp HFOs derive from cortical HFOs. ${ }^{63}$ In this context, it is important to highlight that the skull does not filter high frequencies; it only makes their recording less likely due to the distance and the skull's resistivity, which attenuates an already small activity. For more information, see Zijlmans et al. on how to record HFOs in this issue. ${ }^{3}$

HFO assessment in scalp EEG is a very relevant development, from which a large number of patients could benefit. Applications could include not only gaining additional information on the localization of the epileptic generator, but also assessing disease activity and treatment response, as well as differentiating distinct disease entities. As HFO analysis in scalp EEG is still a relatively young field in HFO research, data on the localizing value of HFOs in scalp EEG are scarce at the moment. Nevertheless, all publications seem to show that HFOs localize to the affected hemisphere or lobe. ${ }^{15,28,64-66}$

That HFOs are able to mirror disease activity has been shown by various authors (see also the sections HFOs Mirror Disease Activity and Important Aspects of HFOs in Children). Pizzo et al. attempted to apply HFO assessment to the differentiation between secondary bilateral synchrony in focal epilepsy and primary bilateral synchrony in idiopathic generalized epilepsy. They found that ripples in secondary bilateral synchrony help to lateralize the epileptic focus. They did not, however, help to differentiate between focal and generalized epilepsy. ${ }^{65}$ Interestingly, the work of Melani et al. ${ }^{66}$ showed that rates of HFOs in the scalp EEG clearly depended on the rates of epileptic spikes. Furthermore, scalp interictal epileptiform discharges, when frequently accompanied by HFOs, were shown to be associated with larger cortical metabolic responses and with thalamic involvement lateralized to the side of cortical ripples. It was proposed that a high rate of epileptic ripples is associated with a more active pathologic cortical-thalamocortical network. ${ }^{67}$

Only very recently, the detection of HFOs $>250 \mathrm{~Hz}$ was attempted in a pilot study. ${ }^{68}$ In this proof-of-principle study, it was shown that it might be feasible to record even frequencies $>250 \mathrm{~Hz}$ with scalp EEG; the rate of fast ripples was considerably lower than that of ripples. Table 1 provides an overview on the literature on HFO assessment in scalp EEG.

\section{$M E G$}

Earlier work investigated the high-frequency content at the time of MEG spikes and at the time of intracranial HFOs in focal epilepsy. ${ }^{69,70}$ Xiang et al. were the first to investigate and source-localize the HF content independently of spikes in children with focal epilepsy. The authors found $\mathrm{HF}$ components between 100 and $1,000 \mathrm{~Hz}$ in $86 \%$ of patients. The loci of this HF activity were concordant with lesions as identified by MRI in $70 \%$ of subjects, and the SOZ as identified by intracranial EEG in $82 \%$ of subjects. ${ }^{16}$ The first study to assess discrete HFO events $>80 \mathrm{~Hz}$ according to HFO definition is from van Klink et al. ${ }^{17}$ The 


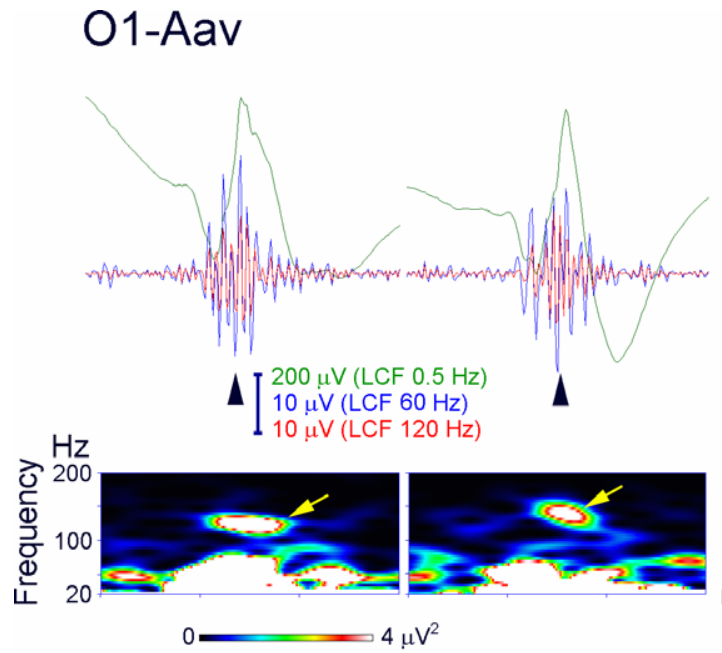

\section{T4-Aav}
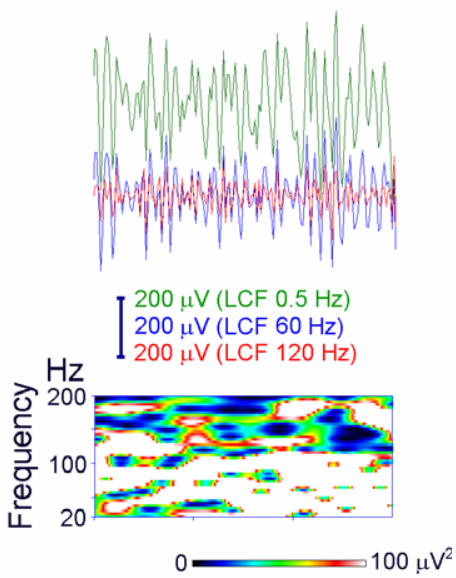

P3-O1

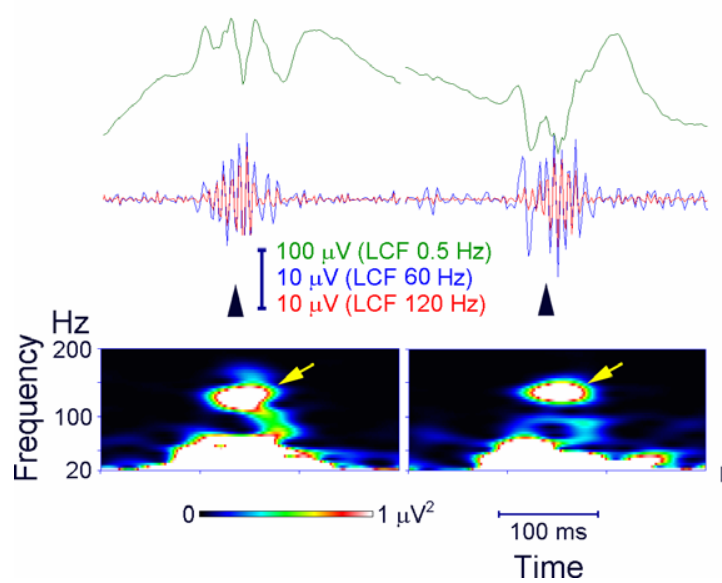

F8-T4
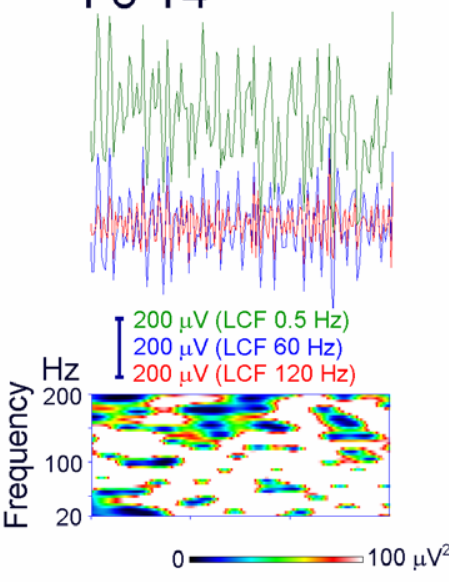

Figure 3.

Ripple oscillations in the scalp electroencephalogram (EEG) recorded from a child with Landau-Kleffner syndrome. Representative spikes (left and middle columns, arrowheads) are associated with ripple oscillation, which was largely invariant irrespective of low-cut frequency (LCF), whether of 60 or $120 \mathrm{~Hz}$ (EEG traces filtered at $0.5,60$, and $120 \mathrm{~Hz}$ are shown in green, blue, and red, respectively). The EEG was recorded during non-rapid eye movement sleep and therefore did not include muscle activity or eye movements. Identical EEG data are presented in a referential montage (top: OI with reference to the average EEG of bilateral earlobes, indicated as OI-Aav) and a bipolar montage (bottom: P3-OI). Note that spike-related ripples with at least four consecutive oscillations are clearly observed in both montages. Each panel of time-frequency spectra shows a corresponding discrete blob (arrows) with a frequency at around I $30 \mathrm{~Hz}$ irrespective of referential or bipolar montage. In contrast, muscle activity (right column) contamination to scalp EEG recorded during wakefulness is dominant over the temporal region (T4, F8-T4) close to muscles and has very irregular morphology and a noisy spectral pattern with no outstanding blobs. Source: Worrell et al., ${ }^{99}$ with permission from Elsevier.

Epilepsia (C) ILAE

authors found ripples in three of 12 patients, and showed an increase of sensitivity to fast oscillations by using virtual channels constructed using beamforming techniques based on information obtained from spikes, thus searching only the brain region generating spikes. Von Ellenrieder et al. showed that fast oscillation events $(40-160 \mathrm{~Hz})$ can be identified correctly independently from information obtained from spikes in MEG. The authors have further demonstrated that it is possible to localize the source of these oscillatory events with high spatial resolution. ${ }^{18}$
Apart from the investigation of interictal HFOs in MEG, two groups focused on the assessment of the ictal HF power in childhood absence epilepsy. ${ }^{71}$ Co-occurring frontal and parietal corticothalamic networks were suggested to interact to produce a pathological state that contributes to the generation of spike and wave discharges. ${ }^{72}$ Interestingly, Tang et al. ${ }^{73}$ demonstrated a correlation between the source strength of ictal HF activity at 200-1,000 Hz and the number of daily seizures in childhood absence epilepsy. These technical developments open now interesting potential 


\begin{tabular}{|c|c|c|c|c|c|c|c|c|}
\hline \multirow[b]{2}{*}{ Authors } & \multirow[b]{2}{*}{ Patients } & \multirow[b]{2}{*}{ Epilepsy type } & \multirow[b]{2}{*}{$\mathrm{N}$} & \multirow[b]{2}{*}{ Method } & \multicolumn{3}{|c|}{$\begin{array}{l}\text { Investigated frequency } \\
\text { band }\end{array}$} & \multirow[b]{2}{*}{ Application } \\
\hline & & & & & Gamma & Ripples & FR & \\
\hline $\begin{array}{l}\text { Kobayashi et al. } \\
(2010)^{14}\end{array}$ & Children & ESES & 10 & $\begin{array}{l}\text { Time frequency plot } \\
\text { and visual }\end{array}$ & & $\mathrm{x}$ & & Proof-of-principle study \\
\hline $\begin{array}{l}\text { Kobayashi et al. } \\
(2011)^{61}\end{array}$ & Children & BCECTS, PS & 32 & $\begin{array}{l}\text { Time frequency plot } \\
\text { and visual }\end{array}$ & & $\mathrm{x}$ & & Prognosis \\
\hline $\begin{array}{l}\text { Andrade-Valenca et al. } \\
(2011)^{15}\end{array}$ & Adults & Focal epilepsy & 15 & Visual & $\mathrm{x}$ & $x$ & & $\begin{array}{l}\text { SOZ identification: accuracy } \\
\text { for gamma, } 70 \% \text {; accuracy } \\
\text { for ripples, } 81 \%\end{array}$ \\
\hline Melani et al. $(2013)^{66}$ & Adults & Focal epilepsy & 32 & Visual & $\mathrm{X}$ & $\mathrm{x}$ & & $\begin{array}{l}\text { Correlation between IED and } \\
\text { gamma and ripple rates }\end{array}$ \\
\hline Fahoum et al. $(20 \mid 4)^{67}$ & Adults & Focal epilepsy & 22 & Visual & $x$ & $x$ & & $\begin{array}{l}\text { Relationship with BOLD } \\
\text { response }\end{array}$ \\
\hline $\begin{array}{l}\text { Kobayashi et al. } \\
(2015)^{55}\end{array}$ & Children & West syndrome & 17 & $\begin{array}{l}\text { Time frequency plot } \\
\text { and visual }\end{array}$ & $x$ & $\mathrm{X}$ & & $\begin{array}{l}\text { Therapy response under } \\
\text { ACTH treatment }\end{array}$ \\
\hline $\begin{array}{l}\text { Chaitanya et al. } \\
(2015)^{74}\end{array}$ & Children & Absence epilepsy & 9 & $\begin{array}{l}\text { ICA, time frequency } \\
\text { analysis }\end{array}$ & & $x$ & & Identification of HFOs \\
\hline Toda et al. $(2015)^{75}$ & Newborns & $\begin{array}{l}\text { Early infantile epileptic } \\
\text { encephalopathy }\end{array}$ & 6 & $\begin{array}{l}\text { Time frequency } \\
\text { analysis }\end{array}$ & & $x$ & & $\begin{array}{l}\text { Presence of HFOs during the } \\
\text { suppression-burst EEG } \\
\text { patterns }\end{array}$ \\
\hline $\begin{array}{l}\text { van Klink et al. } \\
(2016)^{28}\end{array}$ & Adults & Focal epilepsy & 31 & Visual & & $x$ & & $\begin{array}{l}\text { Relationship between ripples } \\
\text { and IEDs }\end{array}$ \\
\hline $\begin{array}{l}\text { van Klink et al. } \\
(2016)^{56}\end{array}$ & Children & Rolandic epilepsy & 22 & Visual & & $x$ & & Prognosis, disease severity \\
\hline Pizzo et al. $(2016)^{65}$ & Adults & GGE and focal epilepsy & 17 & Visual & & $x$ & & Differential diagnosis \\
\hline Pizzo et al. $(2016)^{68}$ & Adults & Focal epilepsy & 10 & Visual & & & $x$ & Proof-of-principle \\
\hline Quian et al. $(2016)^{57}$ & Children & $\begin{array}{l}\text { Atypical benign } \\
\text { partial epilepsy }\end{array}$ & 14 & Visual & & $\mathrm{X}$ & & Severity, treatment response \\
\hline
\end{tabular}

applications for the noninvasive study of fast oscillations with MEG in epilepsy patients.

\section{Important aspects of HFOs in children}

In line with the studies in adults, several retrospective studies using intracranial EEG supported the notion that resection of areas with presurgical high HFO rates is associated with a better postsurgical seizure outcome in children compared to resection of areas with presurgical low HFO rates. 1,40,44 Whereas no difference was found between intracranial rates of HFOs in adults and children, ${ }^{54}$ the rates of scalp HFOs were reported to be up to 100-fold higher in children compared to adults with epilepsy. ${ }^{55}$ Higher HFO rates in the scalp EEG of young children are most likely due to a higher skull conductivity compared to adults. It is therefore not surprising that the number of HFO scalp EEG studies is considerably higher in children compared to adults. Epilepsy syndromes during childhood investigated so far are West syndrome, childhood absence epilepsy, Lennox-Gastaut syndrome, idiopathic location-related epilepsies such as rolandic epilepsy or Panayiotopoulos syndrome, electrical status epilepticus during slow wave sleep, and early infantile epileptic encephalopathy. ${ }^{55-61,74,75}$
A surface EEG study in children with rolandic spikes showed that absence of ripples superimposed on rolandic spikes predicts a benign clinical course, whereas in the presence of several ripples, the child is likely to have more seizures than classical rolandic epilepsy, and pharmacological treatment might be needed. ${ }^{56}$ A scalp HFO study in childhood absence epilepsy also confirmed higher HFO rates during ictal generalized spike-wave discharges compared to interictal generalized spike-wave discharges or sporadic spike-wave discharges. ${ }^{74}$ One study in West syndrome revealed that the rate of HFOs significantly decreased during the course of adrenocorticotropic hormone treatment. ${ }^{55}$ Whether a treatment response with scalp HFOs can also be observed with other antiepileptic treatments awaits future confirmation.

\section{HFOs in Different Types of LESIONS}

There are three studies that aimed at investigating this question. Jacobs et al. selected 12 patients with three types of lesional focal epilepsy (five with unilateral mesial temporal atrophy, four with focal cortical dysplasia, and three with nodular heterotopia). No specific HFO pattern could be 
identified for the different lesion types. ${ }^{76}$ A recent study in a larger sample of 37 patients (13 focal cortical dysplasia, 12 mesial temporal sclerosis, five cortical atrophy, three polymicrogyria, three nodular heterotopia, and one tuberous sclerosis) showed that in patients with intractable epilepsy, the HFO rates vary considerably with different pathologies, and might hence reflect different types of neuronal derangements. Specifically, mesiotemporal lobe sclerosis, focal cortical dysplasia, and nodular heterotopia displayed higher HFO rates compared to polymicrogyria, tuberous sclerosis complex, or atrophy. The authors emphasized the potential usefulness of HFOs as an additional method to better define the extent of the epileptogenic dysplastic tissue in focal cortical dysplasia. ${ }^{77}$ Kerber et al. compared HFO rates in patients with focal cortical dysplasia type I versus type II. Consistent with the literature, patients with focal cortical dysplasia type II had significantly more seizures than those with type I. Interestingly, rates of HFOs were significantly higher in patients with focal cortical dysplasia type II versus type I, suggesting that the activity of HFOs mirrors disease activity. $^{54}$

\section{IMPACT OF SLEEP ON HFOS}

HFOs are influenced by sleep. Following the distribution of epileptic spikes, HFO rates are highest during non-rapid eye movement (NREM) sleep and lowest during rapid eye movement (REM) sleep and wakefulness; these results are independent of the types of electrodes used. Importantly, rates of HFOs were-independently of the sleep stagehigher inside than outside the SOZ.9,78-81

Staba et al. highlighted that ripples decline more drastically compared to fast ripples during REM sleep — a state of maximal desynchronization. This behavior points to fast ripples as the product of pathological neuronal hypersynchronization. ${ }^{9}$ Bagshaw et al. showed that HFOs have their maximal rate in the same sleep stages as the spikes. They also showed that the duration of HFOs is relatively stable across the sleep-wake cycle. ${ }^{79}$ Dümpelmann et al. were interested in examining regional state-specific changes, as most of the previously published evidence is derived from patients with temporal lobe epilepsy. The authors found that HFOs in all brain regions (temporal lobe, rolandic area, parietal lobe, occipital lobe) except the frontal lobe were modulated by sleep. ${ }^{80}$ Clemens et al. ${ }^{78}$ showed that ripple activity increases before spindle peaks and distinctly decreases after the peak.

Recent research focused on the influence of the microstructure of sleep on HFOs. Frauscher et al. ${ }^{82}$ investigated whether the sleep-related activation of HFOs is uniformly distributed across NREM sleep or if it is facilitated by sleep slow oscillations $<1 \mathrm{~Hz}$. These slow oscillations are characterized by a rhythmic alternation between activated (up, when pyramidal cortical neurons are depolarized) and deactivated (down, when pyramidal cortical neurons are hyperpolarized) states, and are shown to influence physiological brain rhythms. Frauscher et al. found that $65 \%$ of ripples occurred during high-amplitude widespread slow waves $<1 \mathrm{~Hz}$ compared to $35 \%$ occurring during control segments of an equal duration. Interestingly, ripples occurred at the transition from the up to the down state, which might underline the role of synchronization, as opposed to hyperexcitability, in the facilitation of HFOs during sleep. Furthermore, HFOs in channels exhibiting epileptic activity or that are part of the SOZ occur during the transition from the up to the following down state, whereas HFOs occurring in channels showing physiological activity occur at the beginning of the next up state. A typical example of this difference in coupling is provided in Figure $4 .^{82}$ In line with this finding, the same group demonstrated that HFOs, known to be suppressed by REM sleep, are even more suppressed during phasic compared to tonic REM sleep; desynchronization is even more increased during phasic compared to tonic REM sleep. ${ }^{83}$ Sakuraba et al. ${ }^{81}$ showed that the suppression of HFOs during REM sleep was less evident inside compared to outside of the epileptogenic zone. The less suppressive effect of REM sleep inside the SOZ may provide a specific marker of epileptogenicity. All studies investigating HFOs across the sleep-wake cycle support the notion that HFO rates are in general higher inside the SOZ compared to outside the SOZ across the different states of vigilance. Assessing HFOs during sleep is advisable, as artifacts are lowest then. Further sleep-specific characteristics such as coupling to slow waves, and the suppressive effect of REM sleep particularly during phasic REM sleep, might help to better delineate the epileptogenic zone and should be further investigated in large multicenter trials.

\section{DIFFERENTIATION BETWEEN Physiological and Pathological HFOs}

One question that has been systematically investigated only recently is how to differentiate physiological ripples, which are thought to reflect summated excitatory postsynaptic potentials, from pathological ripples, which are believed to be slower fast ripples reflecting summated action potentials of synchronously bursting neurons (for more details, see the accompanying review by Jiruska et al. $^{20}$ ). This issue is important, but not easy to address, as rates of ripples vary substantially across different brain areas, as shown by von Ellenrieder et al. ${ }^{84}$ in an investigation of 45 subjects. In humans, physiological HFOs are reported most frequently in the paracentral areas, the hippocampus and the occipital cortex. ${ }^{85-87}$ Investigators attempted to separate physiological from pathological HFOs by considering the coupling with epileptic spikes, ${ }^{26}$ 

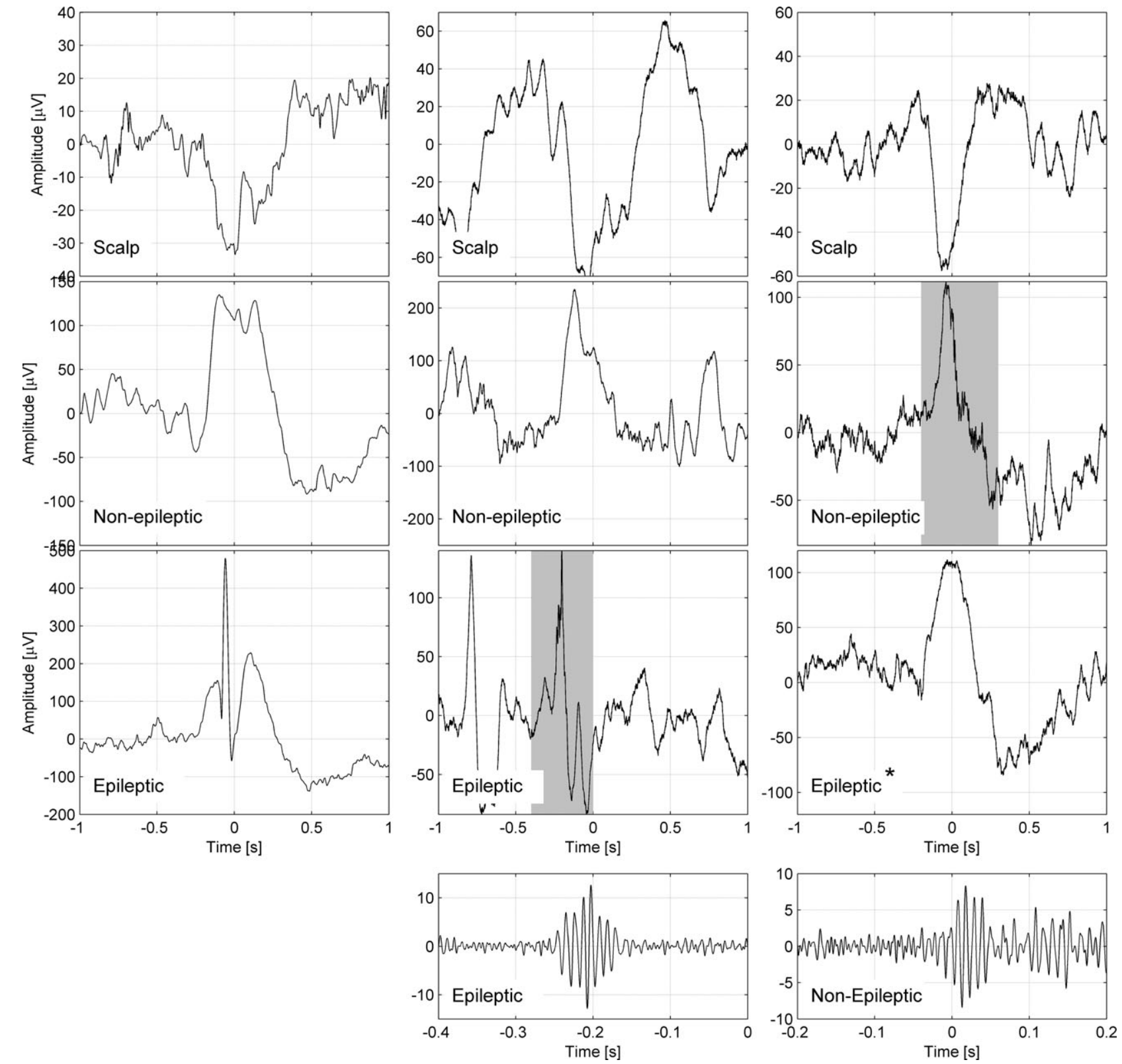

Figure 4.

Representative examples for the coupling of epileptic spikes and high-frequency oscillations (HFOs) across the slow wave cycle. Examples are shown of a slow wave and an epileptic spike (left panel), a slow wave and an HFO in a channel with epileptic activity (middle panel), and an HFO in a channel with normal electroencephalographic (EEG) activity (right panel). The top row shows the slow wave in a scalp channel, the second row shows the same time period for an intracranial channel with normal EEG activity, and the third row shows an intracranial channel with epileptic EEG activity. The fourth row shows the ripple band signal with a different time and amplitude scale, corresponding to the shaded periods in the intracranial channels. All channels are in the left frontal region; each example corresponds to a different patient. The scalp slow wave in the right panel is of shorter duration than the scalp slow waves in the left and middle panels. *In this example, a normal sleep slow wave with no epileptic spike is seen in a channel designated as epileptic because it has spikes at other times. Note that the spike and the HFO in the intracranial channel with epileptic activity (middle) occurs prior to the peak of the scalp negative half-wave, whereas the HFO in the channel with normal EEG activity (right) occurs after the peak of the scalp negative half-wave. Source: Frauscher et al., ${ }^{82}$ published open access using a Creative Commons Attribution CC-BY licence.

Epilepsia (c) ILAE

the background EEG activity, ${ }^{43,86}$ task-induced HFOs, ${ }^{87,88}$ the anatomical location of implanted electrodes, ${ }^{26,86,89}$ the classic features including amplitude, duration, spectral frequency, and rate ${ }^{89,90}$ and the manner of interaction with the accompanying slow wave. ${ }^{82,84,91}$ An overview of the different methods is given in Table 2 . 
Wang et al. showed that neocortical fast ripples and ripples with spikes are specific markers of the SOZ, whereas ripples not going along with spikes are not. The authors concluded that ripples without spikes outside the SOZ may represent spontaneous physiologic ripples in the human neocortex. ${ }^{26}$ Melani et al. described a continuous high-frequency activity, independent of epileptogenicity, which was present almost exclusively in the hippocampus and occipital cortex. They speculated that this continuous activity may be an intrinsic characteristic of specific brain regions, reflecting a particular type of physiological neuronal activity. ${ }^{86}$ In line with this finding, Kerber et al. ${ }^{43}$ suggested that ripples intermixed with an oscillatory background activity may be suggestive of physiological activity, whereas those on a flat background reflect epileptic activity.

Other groups assessed task-induced presumably physiological HFOs using visual tasks, visual motor tasks, and visual memory tasks, ${ }^{87,88}$ or investigated the morphological characteristics of HFOs in epileptic and nonepileptic regions. ${ }^{89,90}$ Despite the different approaches, a clear differentiation of presumably physiological HFOs and pathological epileptic HFOs was not possible, as both types largely overlap with respect to spectral frequency, duration, and amplitude. ${ }^{87-90}$ A similar conclusion was also reached by recent experimental work in the human epileptic subiculum. Alvarado-Rojas et al. ${ }^{92}$ demonstrated that different ripple types have a considerable overlap in spectral frequency despite distinct dynamic changes in inhibition and excitation during interictal and preictal states (see also the review article on fundamental mechanisms of HFOs by Jiruska et al. in this issue ${ }^{20}$ ).

Frauscher et al. ${ }^{82}$ found that HFOs in channels exhibiting interictal epileptiform activity or that are part of the $\mathrm{SOZ}$ occur during the transition from the up to the following down state of the slow wave cycle, whereas presumably physiological HFOs (because they occur in presumably normal brain) occur at the beginning of the next up state. This coupling to the state of the slow wave might therefore help to disentangle physiological from pathological HFOs. This hypothesis was investigated in a larger study using automatic HFO detection by von Ellenrieder et al. ${ }^{84}$ The authors found the association between slow waves and HFOs to be different in normal and epileptic brain regions, emphasizing the different origin of the two HFO types. They also showed that when using this interaction to automatically classify channels as recording from normal/epileptic brain regions, the performance is better than when using other HFO characteristics. ${ }^{84}$ Nonoda et al. investigated the coupling of HFOs to slow waves of $0.5-1 \mathrm{~Hz}$ and 3-4 Hz. The authors found that physiologic ripples generated in stimulationdefined eloquent areas were frequently coupled to the 0.5 $1-\mathrm{Hz}$ slow waves, whereas epileptic ripples generated in the SOZ were coupled to the $3-4-\mathrm{Hz}$ slow waves. ${ }^{91}$ Furthermore, HFOs in channels with physiological activity versus HFOs in channels with epileptic activity were shown to express a different coupling to REM during REM sleep. In

Table 2. Overview of the different methods applied to separate physiological from pathological HFOs

\begin{tabular}{|c|c|c|c|}
\hline Authors & $\mathrm{N}$ & Method & Main finding \\
\hline Nagasawa et al. $(2012)^{87}$ & 10 & Coupling with delta waves & $\begin{array}{l}\text { Epileptic HFOs are coupled with slow waves of } 3-4 \mathrm{~Hz} \text { more } \\
\text { tightly compared to physiological HFOs, which are coupled to } \\
\text { slow waves of } 0.5-1 \mathrm{~Hz}\end{array}$ \\
\hline Matsumoto et al. $(2013)^{88}$ & 5 & $\begin{array}{l}\text { Comparison of task-induced HFOs } \\
\text { vs. spontaneous HFOs }\end{array}$ & $\begin{array}{l}\text { Substantial overlap in all classical features between task-induced } \\
\text { and spontaneous HFOs }\end{array}$ \\
\hline Melani et al. $(2013)^{86}$ & 22 & Evaluation of background EEG & Continuous high-frequency activity as a physiological pattern \\
\hline Wang et al. $(2013)^{26}$ & 35 & $\begin{array}{l}\text { Ripples with accompanying epileptic } \\
\text { spike vs. isolated ripples }\end{array}$ & $\begin{array}{l}\text { Ripples associated with spikes are more specific for the SOZ } \\
\text { compared to ripples not co-occurring with spikes }\end{array}$ \\
\hline Alkawadri et al. $(2014)^{89}$ & 7 & $\begin{array}{l}\text { Differences in classical features }{ }^{\mathrm{a}} \\
\text { between epileptic and nonepileptic } \\
\text { brain regions }\end{array}$ & $\begin{array}{l}\text { Substantial overlap in all classical features between the normal } \\
\text { and the epileptic zone }\end{array}$ \\
\hline Kerber et al. $(2014)^{43}$ & 32 & Evaluation of background EEG & $\begin{array}{l}\text { HFOs on flat background are more specific for SOZ than HFOs } \\
\text { on oscillatory background }\end{array}$ \\
\hline Frauscher et al. $(2015)^{82}$ & 8 & Coupling with slow waves & $\begin{array}{l}\text { Different coupling of physiological and pathological HFOs in } \\
\text { relation to slow waves }\end{array}$ \\
\hline Malinowska et al. $(2015)^{90}$ & 33 & $\begin{array}{l}\text { Differentiation in classical features }{ }^{\mathrm{a}} \\
\text { between SOZ and non-SOZ }\end{array}$ & $\begin{array}{l}\text { Significant differences in classical features, however, substantial } \\
\text { overlap }\end{array}$ \\
\hline Von Ellenrieder et al. $(2016)^{84}$ & 45 & Coupling with slow waves & $\begin{array}{l}\text { Interaction with slow waves during sleep improves } \\
\text { discrimination of physiologic and pathologic HFOs }\end{array}$ \\
\hline Frauscher et al. $(2016)^{83}$ & 12 & Coupling with REMs & $\begin{array}{l}\text { Different coupling of physiological and pathological HFOs in } \\
\text { relation to REMs during REM sleep }\end{array}$ \\
\hline Nonoda et al. $(2016)^{91}$ & 13 & $\begin{array}{l}\text { Coupling with different frequencies of } \\
\text { slow waves }\end{array}$ & $\begin{array}{l}\text { Epileptic HFOs are coupled with slow waves of } 3-4 \mathrm{~Hz} \text { more } \\
\text { tightly compared to physiological HFOs, which are coupled to } \\
\text { slow waves of } 0.5-1 \mathrm{~Hz}\end{array}$ \\
\hline
\end{tabular}


contrast to ripples in the $\mathrm{SOZ}$ or exclusively irritative zone, physiologic ripples were more abundant during phasic REM sleep. ${ }^{83}$ It may therefore be more important to know the EEG context of occurrence of an HFO than its morphology to determine whether it is normal or pathological.

\section{Visual Versus Automatic DETECTION OF HFOs}

Visual analysis of HFOs is considered to be the gold standard for HFO assessment. Visual analysis, however, entails serious obstacles making HFO assessment impossible for clinical routine; visual marking of HFOs is very time-consuming, requires expertise, and might be subjective if an interrater agreement is not sought. To overcome these drawbacks, various detectors were developed and validated over recent years (for more details, see Zijlmans et $\mathrm{al}^{3}{ }^{3}$ ). Automated and visual detection of HFOs yield comparable identification of the $\mathrm{SOZ}^{93}$; a meta-analysis showed that removal of automatically detected HFOs and visually detected HFOs in presurgical data yield similar results with respect to good surgical outcome. ${ }^{48}$

Unfortunately, it is not possible to directly compare sensitivity and specificity between the publications on automated detection of HFOs, because most algorithms depend on many parameters that need to be tuned and on the ground truth used, which is again subjective visual identification. Existing comparison studies which were based on the same ground truth data showed that there are differences when applying the different detectors. Nevertheless, the ranking of channels with high versus low HFO rates is comparatively consistent. ${ }^{94}$ In addition, it seems that sensitivity of algorithms is not the most important criterion. Removal of regions with a high rate of HFO occurrence is more strongly related to good surgical outcome than removal of all HFOgenerating tissue. ${ }^{48}$

One aspect that is very important when using automatic HFO detection is artifact removal. Whereas some detectors are semiautomatic, requiring visual validation, others have implemented fully automated postprocessing steps for artifact removal, so that the detector can be used to work autonomously for large datasets as needed in clinical routine. The general validity of this approach has not been established.

Finally, to make the results of these technical developments useful for clinical practice, they need to be implemented in software that satisfies the needs of clinical routine.

\section{Future Directions}

Increasing evidence has been collected over the past decade underlining that HFOs might be a promising biomarker for the epileptogenic zone, and that removal of regions with high HFO rates is associated with a good postsurgical outcome. All existing data are derived from comparatively small retrospective studies. No study so far was adequately powered and methodologically designed to investigate the probably more important clinical question of whether a patient is more likely to become seizure-free if the HFO resection ratio is high than if HFO-generating regions are not resected. There is therefore a need for properly designed, high-quality, adequately powered, randomized multicenter trials to determine whether interictal HFOs are true markers for the epileptogenic zone. One such trial is currently underway in The Netherlands for intraoperative electrocorticography. ${ }^{95}$ In this trial, surgery is tailored by HFOs (arm 1) or interictal spikes (arm 2) in the intraoperative electrocorticography. The trial has a noninferiority design to test feasibility and at least equal performance in terms of surgical outcome. If this trial is positive, future multicenter collaborations with large sample sizes using a superiority design and objective easy HFO analysis to standardize HFO assessment are warranted to demonstrate that the use of HFOs as a biomarker for tailoring will increase the success rate of epilepsy surgery while reducing resection volume, potentially leading to a reduction of neurological deficits and better quality of life.

It will also be interesting to compare HFO detection with other approaches for ictal determination of the epileptogenic zone. Recent methods were introduced to quantify the presumed degree of epileptogenicity of brain structures recorded by depth-EEG electrodes ${ }^{37,96-}$ 98 for comparative study of quantitative methods. Moreover, it will be of interest to translate the knowledge gathered from experimental work indicating that different HFO seizure onset patterns are expressions of different mechanisms of epileptogenesis (see the accompanying paper by Jiruska et al. ${ }^{20}$ in this issue for more information). This knowledge might help to identify more efficacious antiepileptic strategies tailored directly to the underlying mechanisms.

Although there is no class I evidence for the use of HFOs yet, evidence is accumulating, and as in many other fields where class I evidence is lacking, this accumulation of concordant evidence indicates that it may be time to start implementing HFOs in clinical patient care. Particularly the assessment of HFOs using noninvasive methods will open a new avenue allowing not only to identify the SOZ, but also to assess disease activity in large numbers of patients.

\section{ACKnowledgments}

This publication is the outcome of the 2nd International Workshop on High Frequency Oscillations in Epilepsy held in Freiburg on March 10-12, 2016. The workshop was supported by the German Research Foundation (grant DFG JA1725/3-1).

\section{Disclosure}

The authors declare no conflicts of interest. The authors confirm that they have read the Journal's position on issues involved in ethical publication and affirm that this report is consistent with those guidelines. 


\section{REFERENCES}

1. Jacobs J, Staba R, Asano E, et al. High-frequency oscillations (HFOs) in clinical epilepsy. Prog Neurobiol 2012;98:302-315.

2. Bragin A, Engel J Jr, Wilson CL, et al. High-frequency oscillations in human brain. Hippocampus 1999;9:137-142.

3. Zijlmans M, Worrell GA, Dümpelmann M. How to record high-frequency oscillations in epilepsy: A practical guideline. Epilepsia; 2017:58. https://doi.org/10.1111/epi.13814

4. Allen PJ, Fish DR, Smith SJ. Very high-frequency rhythmic activity during SEEG suppression in frontal lobe epilepsy. Electroencephalogr Clin Neurophysiol 1992;82:155-159.

5. Fisher RS, Webber WR, Lesser RP, et al. High-frequency EEG activity at the start of seizures. J Clin Neurophysiol 1992;9:441-448.

6. Bragin A, Engel J Jr, Wilson CL, et al. Hippocampal and entorhinal cortex high-frequency oscillations $(100-500 \mathrm{~Hz})$ in human epileptic brain and in kainic acid-treated rats with chronic seizures. Epilepsia 1999;40:127-137.

7. Bragin A, Engel J Jr, Wilson CL, et al. Electrophysiologic analysis of a chronic seizure model after unilateral hippocampal KA injection. Epilepsia 1999;40:1210-1221.

8. Bragin A, Wilson CL, Staba RJ, et al. Interictal high-frequency oscillations $(80-500 \mathrm{~Hz})$ in the human epileptic brain: entorhinal cortex. Ann Neurol 2002;52:407-415.

9. Staba RJ, Wilson CL, Bragin A, et al. High-frequency oscillations recorded in human medial temporal lobe during sleep. Ann Neurol 2004;56:108-115.

10. Jirsch JD, Urrestarazu E, LeVan P, et al. High-frequency oscillations during human focal seizures. Brain 2006;129:1593-1608.

11. Urrestarazu E, Jirsch JD, LeVan P, et al. High-frequency intracerebral EEG activity $(100-500 \mathrm{~Hz})$ following interictal spikes. Epilepsia 2006; 47:1465-1476.

12. Akiyama T, Otsubo H, Ochi A, et al. Focal cortical high-frequency oscillations trigger epileptic spasms: confirmation by digital video subdural EEG. Clin Neurophysiol 2005;116:2819-2825.

13. Châtillon CÉ, Zelmann R, Bortel A, et al. Contact size does not affect high frequency oscillation detection in intracerebral EEG recordings in a rat epilepsy model. Clin Neurophysiol 2011;122:1701-1705.

14. Kobayashi K, Watanabe Y, Inoue T, et al. Scalp-recorded high-frequency oscillations in childhood sleep-induced electrical status epilepticus. Epilepsia 2010;51:2190-2194.

15. Andrade-Valenca LP, Dubeau F, Mari F, et al. Interictal scalp fast oscillations as a marker of the seizure onset zone. Neurology 2011;77:524-531.

16. Xiang J, Liu Y, Wang Y, et al. Frequency and spatial characteristics of high-frequency neuromagnetic signals in childhood epilepsy. Epileptic Disord 2009;11:113-125.

17. van Klink N, Hillebrand A, Zijlmans M. Identification of epileptic high frequency oscillations in the time domain by using MEG beamformerbased virtual sensors. Clin Neurophysiol 2016;127:197-208.

18. von Ellenrieder N, Pellegrino G, Hedrich T, et al. Detection and magnetic source imaging of fast oscillations $(40-160 \mathrm{~Hz})$ recorded with magnetoencephalography in focal epilepsy patients. Brain Topogr 2016;29:218-231

19. Urrestarazu E, Chander R, Dubeau F, et al. Interictal high-frequency oscillations $(100-500 \mathrm{~Hz})$ in the intracerebral EEG of epileptic patients. Brain 2007;130:2354-2366.

20. Jiruska P, Alvarado-Rojas C, Schevon CA, et al. Update on the mechanisms and roles of high-frequency oscillations in seizures and epileptic disorders. Epilepsia 2017. https://doi.org/10.1111/epi.13830

21. Khosravani H, Mehrotra N, Rigby M, et al. Spatial localization and time-dependant changes of electrographic high frequency oscillations in human temporal lobe epilepsy. Epilepsia 2009;50:605-616.

22. Zijlmans M, Jacobs J, Zelmann R, et al. High-frequency oscillations mirror disease activity in patients with epilepsy. Neurology 2009;72:979-986.

23. Gotman J, Marciani MG. Electroencephalographic spiking activity, drug levels, and seizure occurrence in epileptic patients. Ann Neurol 1985; 17:597-603

24. Goncharova II, Alkawadri R, Gaspard N, et al. The relationship between seizures, interictal spikes and antiepileptic drugs. Clin Neurophysiol 2016;127:3180-3186.

25. Jacobs J, LeVan P, Chander R, et al. Interictal high-frequency oscillations $(80-500 \mathrm{~Hz})$ are an indicator of seizure onset areas independent of spikes in the human epileptic brain. Epilepsia 2008;49:1893-1907.
26. Wang S, Wang IZ, Bulacio JC, et al. Ripple classification helps to localize the seizure-onset zone in neocortical epilepsy. Epilepsia 2013;54:370-376.

27. Bénar CG, Chauvière L, Bartolomei F, et al. Pitfalls of high-pass filtering for detecting epileptic oscillations: a technical note on "false" ripples. Clin Neurophysiol 2010;121:301-310.

28. van Klink N, Frauscher B, Zijlmans M, et al. Relationships between interictal epileptic spikes and ripples in surface EEG. Clin Neurophysiol 2016;127:143-149.

29. Jacobs J, Vogt C, LeVan P, et al. The identification of distinct high-frequency oscillations during spikes delineates the seizure onset zone better than high-frequency spectral power changes. Clin Neurophysiol 2016;127:129-142.

30. Burnos S, Frauscher B, Zelmann R, et al. The morphology of high frequency oscillations (HFO) does not improve delineating the epileptogenic zone. Clin Neurophysiol 2016;127:2140-2148.

31. Jacobs J, Zelmann R, Jirsch J, et al. High frequency oscillations (80$500 \mathrm{~Hz}$ ) in the preictal period in patients with focal seizures. Epilepsia 2009;50:1780-1792.

32. Sato Y, Doesburg SM, Wong SM, et al. Preictal surrender of post-spike slow waves to spike-related high-frequency oscillations $(80-200 \mathrm{~Hz})$ is associated with seizure initiation. Epilepsia 2014;55:1399-1405.

33. Sato Y, Doesburg SM, Wong SM, et al. Dynamic preictal relations in FCD type II: potential for early seizure detection in focal epilepsy. Epilepsy Res 2015;110:26-31.

34. Zijlmans M, Jacobs J, Kahn YU, et al. Ictal and interictal high frequency oscillations in patients with focal epilepsy. Clin Neurophysiol 2011;122:664-671.

35. Akiyama T, Chan DW, Go CY, et al. Topographic movie of intracranial ictal high-frequency oscillations with seizure semiology: epileptic network in Jacksonian seizures. Epilepsia 2011;52:75-83.

36. Usui N, Terada K, Baba K, et al. Significance of very-high-frequency oscillations (over 1,000 Hz) in epilepsy. Ann Neurol 2015;78:295-302.

37. David O, Blauwblomme T, Job AS, et al. Imaging the seizure onset zone with stereo-electroencephalography. Brain 2011;134:2898-2911.

38. Usui N, Terada K, Baba K, et al. Clinical significance of ictal high frequency oscillations in medial temporal lobe epilepsy. Clin Neurophysiol 2011;122:1693-1700.

39. van 't Klooster MA, Zijlmans M, Leijten FS, et al. Time-frequency analysis of single pulse electrical stimulation to assist delineation of epileptogenic cortex. Brain 2011;134:2855-2866.

40. Fujiwara H, Greiner HM, Lee KH, et al. Resection of ictal high-frequency oscillations leads to favorable surgical outcome in pediatric epilepsy. Epilepsia 2012;53:1607-1617.

41. Haegelen C, Perucca P, Châtillon CE, et al. High-frequency oscillations, extent of surgical resection, and surgical outcome in drug-resistant focal epilepsy. Epilepsia 2013;54:848-857.

42. Cho JR, Koo DL, Joo EY, et al. Resection of individually identified high-rate high-frequency oscillations region is associated with favorable outcome in neocortical epilepsy. Epilepsia 2014;55:1872-1883.

43. Kerber K, Dümpelmann M, Schelter B, et al. Differentiation of specific ripple patterns helps to identify epileptogenic areas for surgical procedures. Clin Neurophysiol 2014;125:1339-1345.

44. Okanishi T, Akiyama T, Tanaka S, et al. Interictal high frequency oscillations correlating with seizure outcome in patients with widespread epileptic networks in tuberous sclerosis complex. Epilepsia 2014;55:1602-1610.

45. van Klink NE, Van 't Klooster MA, Zelmann R, et al. High frequency oscillations in intra-operative electrocorticography before and after epilepsy surgery. Clin Neurophysiol 2014;125:2212-2219.

46. van 't Klooster MA, van Klink NE, Leijten FS, et al. Residual fast ripples in the intraoperative corticogram predict epilepsy surgery outcome. Neurology 2015;85:120-128.

47. van 't Klooster MA, Van Klink NEC, Zweiphenning WJEM, et al. Tailoring epilepsy surgery with fast ripples in the intra-operative electrocorticogram. Ann Neurol 2017;81:664-676.

48. Höller Y, Kutil R, Klaffenboeck L, et al. High-frequency oscillations in epilepsy and surgical outcome. A meta-analysis. Front Hum Neurosci 2015;9:574.

49. Gloss D, Nolan SJ, Staba R. The role of high-frequency oscillations in epilepsy surgery planning. Cochrane Database Syst Rev 2014;(1):CD010235.

50. Bragin A, Wilson CL, Almajano J, et al. High-frequency oscillations after status epilepticus: epileptogenesis and seizure genesis. Epilepsia 2004;45:1017-1023. 
51. Zijlmans M, Jacobs J, Zelmann R, et al. High frequency oscillations and seizure frequency in patients with focal epilepsy. Epilepsy Res 2009;85:287-292.

52. Zijlmans M, Huiskamp GM, Cremer OL, et al. Epileptic high-frequency oscillations in intraoperative electrocorticography: the effect of propofol. Epilepsia 2012;53:1799-1809.

53. Rampp S, Schmitt HJ, Heers M, et al. Etomidate activates epileptic high frequency oscillations. Clin Neurophysiol 2014;125:223-230.

54. Kerber K, LeVan P, Dümpelmann M, et al. High frequency oscillations mirror disease activity in patients with focal cortical dysplasia. Epilepsia 2013;54:1428-1436.

55. Kobayashi K, Akiyama T, Oka M, et al. A storm of fast $(40-150 \mathrm{~Hz})$ oscillations during hypsarrhythmia in West syndrome. Ann Neurol 2015;77:58-67.

56. van Klink N, van 't Klooster M, Leijten F, et al. Ripples on rolandic spikes: a marker of epilepsy severity. Epilepsia 2016;57:1179-1189.

57. Quian P, Li H, Xue J, et al. Scalp-recorded high-frequency oscillations in atypical benign partial epilepsy. Clin Neurophysiol 2016;127:3306-3313.

58. Kobayashi K, Oka M, Akiyama T, et al. Very fast rhythmic activity on scalp EEG associated with epileptic spasms. Epilepsia 2004;45:488-496.

59. Inoue T, Kobayashi K, Oka M, et al. Spectral characteristics of EEG gamma rhythms associated with epileptic spasms. Brain Dev 2008;30:321-328.

60. Kobayashi K, Inoue T, Watanabe Y, et al. Spectral analysis of EEG gamma rhythms associated with tonic seizures in Lennox-Gastaut syndrome. Epilepsy Res 2009;86:15-22.

61. Kobayashi K, Yoshinaga H, Toda Y, et al. High-frequency oscillations in idiopathic partial epilepsy of childhood. Epilepsia 2011;52:18121819.

62. von Ellenrieder N, Beltrachini L, Perucca P, et al. Size of cortical generators of epileptic interictal events and visibility on scalp EEG. Neuroimage 2014;94:47-54.

63. Zelmann R, Lina JM, Schulze-Bonhage A, et al. Scalp EEG is not a blur: it can see high frequency oscillations although their generators are small. Brain Topogr 2014;27:683-704.

64. Iwatani Y, Kagitani-Shimono K, Tominaga K, et al. Ictal high-frequency oscillations on scalp EEG recordings in symptomatic West syndrome. Epilepsy Res 2012;102:60-70.

65. Pizzo F, Ferrari-Marinho T, Amiri M, et al. When spikes are symmetric, ripples are not: bilateral spike and wave above $80 \mathrm{~Hz}$ in focal and generalized epilepsy. Clin Neurophysiol 2016;127:1794-1802.

66. Melani F, Zelmann R, Dubeau F, et al. Occurrence of scalp-fast oscillations among patients with different spiking rate and their role as epileptogenicity marker. Epilepsy Res 2013;106:345-356.

67. Fahoum F, Melani F, Andrade-Valença L, et al. Epileptic scalp ripples are associated with corticothalamic BOLD changes. Epilepsia 2014;55:1611-1619.

68. Pizzo F, Frauscher B, Ferrari-Marinho T, et al. Detectability of fast ripples $(>250 \mathrm{~Hz})$ on the scalp EEG: a proof-of-principle study with subdermal electrodes. Brain Topogr 2016;29:358-367.

69. Guggisberg AG, Kirsch HE, Mantle MM, et al. Fast oscillations associated with interictal spikes localize the epileptogenic zone in patients with partial epilepsy. Neuroimage 2008;39:661-668.

70. Rampp S, Kaltenhäuser M, Weigel D, et al. MEG correlates of epileptic high gamma oscillations in invasive EEG. Epilepsia 2010;51:1638-1642.

71. Miao A, Tang L, Xiang J, et al. Dynamic magnetic source imaging of absence seizure initialization and propagation: a magnetoencephalography study. Epilepsy Res 2014;108:468-480.

72. Tenney JR, Fujiwara H, Horn PS, et al. Low- and high-frequency oscillations reveal distinct absence seizure networks. Ann Neurol 2014;76:558-567.

73. Tang L, Xiang J, Huang S, et al. Neuromagnetic high-frequency oscillations correlate with seizure severity in absence epilepsy. Clin Neurophysiol 2016;127:1120-1129.

74. Chaitanya G, Sinha S, Narayanan M, et al. Scalp high frequency oscillations (HFOs) in absence epilepsy: an independent component analysis (ICA) based approach. Epilepsy Res 2015;115:133-140.

75. Toda Y, Kobayashi K, Hayashi Y, et al. High-frequency EEG activity in epileptic encephalopathy with suppression-burst. Brain Dev 2015;37:230-236.

76. Jacobs J, Levan P, Châtillon CE, et al. High frequency oscillations in intracranial EEGs mark epileptogenicity rather than lesion type. Brain 2009;132:1022-1037.
77. Ferrari-Marinho T, Perruca P, Mok K, et al. Pathological substrates of focal epilepsy influence the generation of high-frequency oscillations. Epilepsia 2015;56:592-598.

78. Clemens Z, Mölle M, Eross L, et al. Temporal coupling of parahippocampal ripples, sleep spindles and slow oscillations in humans. Brain 2007; 130:2868-2878.

79. Bagshaw AP, Jacobs J, LeVan P, et al. Effect of sleep stage on interictal high-frequency oscillations recorded from depth macroelectrodes in patients with focal epilepsy. Epilepsia 2009;50:617-628.

80. Dümpelmann M, Jacobs J, Schulze-Bonhage A. Temporal and spatial characteristics of high frequency oscillations as a new biomarker in epilepsy. Epilepsia 2015;56:197-206.

81. Sakuraba R, Iwasaki M, Okumura E, et al. High frequency oscillations are less frequent but more specific to epileptogenicity during rapid eye movement sleep. Clin Neurophysiol 2016;127:179-186.

82. Frauscher B, von Ellenrieder N, Ferrari-Marinho T, et al. Facilitation of epileptic activity during sleep is mediated by high amplitude slow waves. Brain 2015;138:1629-1641.

83. Frauscher B, von Ellenrieder N, Dubeau F, et al. EEG desynchronization during phasic REM sleep suppresses interictal epileptic activity in humans. Epilepsia 2016;57:879-888.

84. von Ellenrieder N, Frauscher B, Dubeau F, et al. Interaction with slow waves during sleep improves discrimination of physiological and pathological high frequency oscillations $(80-500 \mathrm{~Hz})$. Epilepsia 2016;57:869-878.

85. Axmacher N, Elger CE, Fell J. Ripples in the medial temporal lobe are relevant for human memory consolidation. Brain 2008;131:1806-1817.

86. Melani F, Zelmann R, Mari F, et al. Continuous high frequency activity: a peculiar SEEG pattern related to specific brain regions. $\mathrm{Clin} \mathrm{Neu}-$ rophysiol 2013;124:1507-1516.

87. Nagasawa T, Juhasz C, Rothermel R, et al. Spontaneous and visuallydriven high-frequency oscillations in the occipital cortex: intracranial recordings in epileptic patients. Hum Brain Mapp 2012;33:569-583.

88. Matsumoto A, Brinkmann BH, Matthew Stead S, et al. Pathological and physiological high-frequency oscillations in focal human epilepsy. J Neurophysiol 2013;110:1958-1964.

89. Alkawadri R, Gaspard N, Goncharaova II, et al. The spatial and signal characteristics of physiological high frequency oscillations. Epilepsia 2014;55:1986-1995.

90. Malinowska U, Bergey GK, Harezlak J, et al. Identification of seizure onset zone and preictal state based on characteristics of high frequency oscillations. Clin Neurophysiol 2015;126:1505-1513.

91. Nonoda Y, Miyakoshi M, Ojeda A, et al. Interictal high-frequency oscillations generated by seizure onset and eloquent areas may be differentially coupled with different slow waves. Clin Neurophysiol 2016;127:2489-2499.

92. Alvarado-Rojas C, Huberfeld G, Baulac M, et al. Different mechanisms of ripple-like oscillations in the human epileptic subiculum. Ann Neurol 2015;77:281-290.

93. Pail M, Halámek J, Daniel P, et al. Intracerebrally recorded high frequency oscillations: simple visual assessment versus automated detection. Clin Neurophysiol 2013;124:1935-1942.

94. Zelmann R, Mari F, Jacobs J, et al. A comparison between detectors of high frequency oscillations. Clin Neurophysiol 2012;123:106-116.

95. van 't Klooster MA, Leijten FS, Huiskamp G, et al. High frequency oscillations in the intra-operative ECoG to guide epilepsy surgery ("The HFO Trial"): study protocol for a randomized controlled trial. Trials 2015; $16: 422$.

96. Gnatkovsky V, Francione S, Cardinale F, et al. Identification of reproducible ictal patterns based on quantified frequency analysis of intracranial EEG signals. Epilepsia 2011;52:477-488.

97. Andrzejak RG, David O, Gnatkovsky V, et al. Localization of epileptogenic zone on pre-surgical intracranial EEG recordings: toward a validation of quantitative signal analysis approaches. Brain Topogr 2015;28:832-837.

98. Bartolomei F, Chauvel P, Wendling F. Epileptogenicity of brain structures in human temporal lobe epilepsy: a quantified study from intracerebral EEG. Brain 2008;131:1818-1830.

99. Worrell GA, Jerbi K, Kobayashi K, et al. Recording and analysis techniques for high-frequency oscillations. Prog Neurobiol 2012;98:265278. 